\title{
DETERMINAÇÃO DA CONDUTIVIDADE TÉRMICA EFETIVA NA ESTAGNAÇÃO DE SISTEMAS MONODISPERSOS PELO MÉTODO DA SONDA LINEAR
}

\author{
É. F. R. TADA ${ }^{1}$, V. F. BERTUCCI ${ }^{1}$ e J. C. THOMÉO ${ }^{1}$ \\ ${ }^{1}$ Universidade Estadual Paulista, Departamento de Engenharia e Tecnologia de Alimentos \\ E-mail para contato: erikartada@gmail.com
}

\begin{abstract}
RESUMO - A condutividade térmica efetiva na estagnação foi determinada através do método da sonda linear como uma função da temperatura para leitos secos e inertes compostos por esferas de vidro e esferas de alumina. Para ambos os leitos de partículas, o parâmetro apresentou diferença significativa a $50^{\circ} \mathrm{C}$, em relação às demais temperaturas analisadas. Foram propostas relações da condutividade térmica na estagnação como uma função da temperatura para os leitos empregados. O modelo de resistências em paralelo foi utilizado para estimativa teórica do parâmetro e apresentou proximidade com os dados experimentais.
\end{abstract}

\section{INTRODUÇÃO}

Um meio poroso pode ser entendido como aquele constituído por duas fases, uma sólida e outra fluida (Tsotsas; Martin, 1987). Diversos processos envolvendo meios porosos vêm sendo estudados a partir de modelagem e simulação (Boateng; Barr, 1996; Fanaei e Vaziri, 2009; Wang et al., 2010; Casciatori et al., 2016; Tada et al., 2017).

Os fenômenos que ocorrem em meios porosos podem ser representados através de modelos a uma fase, também chamados de pseudo-homogêneos, ou de modelos a duas fases. Em modelos pseudo-homogêneos, as propriedades do leito são calculadas com base nas propriedades individuais de cada fase e considera-se que as fases que o constitui encontram-se em equilíbrio. Assim, o meio poroso é visto como um sólido e um único equacionamento é necessário para a representação dos fenômenos. Já em modelos a duas fases, considera-se a existência das fases e a interação entre as mesmas. Equacionamentos independentes são necessários para cada uma das fases e o nível de complexidade destes modelos é maior, pois requerem uma maior quantidade de parâmetros e termos de representação. Apesar da complexidade, modelos a duas fases são capazes de prever fenômenos limitados aos modelos pseudo-homogêneos (THOMÉO, 1990; THOMÉO, FREIRE, 2009).

A condutividade térmica efetiva na estagnação $\left(K_{0}\right)$ representa a facilidade de propagação de calor em um meio, e é uma das propriedades requeridas por modelos a uma fase para transferência de calor. Dentre os métodos desenvolvidos para determinação experimental desta propriedade, o método da sonda linear requer simples aparato experimental, pouco tempo e apresenta resultados relativamente confiáveis. Este método foi desenvolvido em 1949 por Van der Held e Van Drunen, e baseia-se na propagação de calor 
fornecido por uma fonte infinitesimal através de um meio homogêneo infinito. Modelos teóricos também são reportados pela literatura para estimativa de propriedades de meios porosos, tais como o modelo de resistências em paralelo de Goedeken et al. (1998) e equações de Choi e Okos (1986). O primeiro baseia-se na soma ponderada das propriedades de cada fase que constitui um meio poroso e o segundo, em relações específicas de composição do meio. Estes equacionamentos têm sido amplamente empregados na estimativa de materiais heterogêneos (Neckel; Mariani, 2010; Casciatori et al., 2013; Tada et al., 2017), e a literatura reporta boa concordância dos dados estimados com resultados obtidos através do método da sonda linear (Casciatori et al., 2013).

Neste contexto, o presente trabalho tem como objetivo a determinação da condutividade térmica efetiva na estagnação de partículas regulares secas em diferentes temperaturas através do método da sonda linear, bem como a comparação dos resultados obtidos com dados estimados através do modelo de resistências em paralelo.

\section{MATERIAIS E MÉTODOS}

\subsection{Materiais}

Esferas de vidro e esferas de alumina de $3 \mathrm{~mm}$ de diâmetro médio foram empregadas nos ensaios para determinação da condutividade térmica efetiva na estagnação $\left(\mathrm{K}_{0}\right)$ de sistemas monodispersos. A escolha dos materiais foi motivada pelo fato de que as partículas de vidro são impermeáveis e as partículas de alumina possuem capacidade hidroscópica, de modo que as próprias partículas possuem poros em seu interior.

\subsection{Aparato experimental e ensaios para determinação de $\mathbf{K}_{0}$}

O conjunto para determinação de $K_{0}$ consistiu em um cilindro encamisado com $9.5 \mathrm{~cm}$ de diâmetro interno e $24,1 \mathrm{~cm}$ de comprimento construído em aço inoxidável. Através da camisa, circulou água em temperatura controlada, fornecida por um banho termostático. No centro radial do cilindro interno, mantinha-se um fio isolado eletricamente por óxido de magnésio e um termopar do tipo $\mathrm{T}$, ambos envoltos por aço inoxidável e compondo um cilindro com $6 \mathrm{~mm}$ de diâmetro externo e 21,5 cm de comprimento. Este fio foi conectado a uma fonte de corrente contínua, de modo que a energia fornecida para o sistema através do fio foi previamente conhecida. Tampas de $1,5 \mathrm{~cm}$ de espessura foram inseridas nas extremidades axiais do conjunto. Durante os ensaios, o conjunto foi mantido imerso em banho termostático em temperatura controlada.

As partículas foram inseridas neste sistema através de uma das extremidades axiais. Termopares do tipo $\mathrm{T}$ foram inseridos ao longo do raio para monitoramento da temperatura da amostra. Os sinais emitidos pelos termopares foram adquiridos e armazenados por um sistema de aquisição de dados COMPAQ-DAQ ${ }^{\circledR}$ (National Instruments, Austin, USA) gerenciado por uma rotina do software LabView ${ }^{\circledR}$ (National Instruments, Austin, USA). Quando atingido o equilíbrio térmico entre a amostra e a temperatura externa, o fio no centro do conjunto foi energizado e o aumento de temperatura ao longo do tempo foi observado. Os ensaios foram conduzidos para as temperaturas $25,37.5$ e $50^{\circ} \mathrm{C}$, em triplicata, e os resultados foram submetidos à análise de variância ANOVA. Em caso de diferença significativa, os resultados foram analisados estatisticamente segundo teste de comparação de médias a $5 \%$ de 
significância. Na Figura 1 está apresentado um diagrama esquemático do conjunto utilizado para estes ensaios, extraído de Casciatori et al. (2013).

Figura 1 - Aparato experimental utilizado para determinação da condutividade térmica efetiva na estagnação.

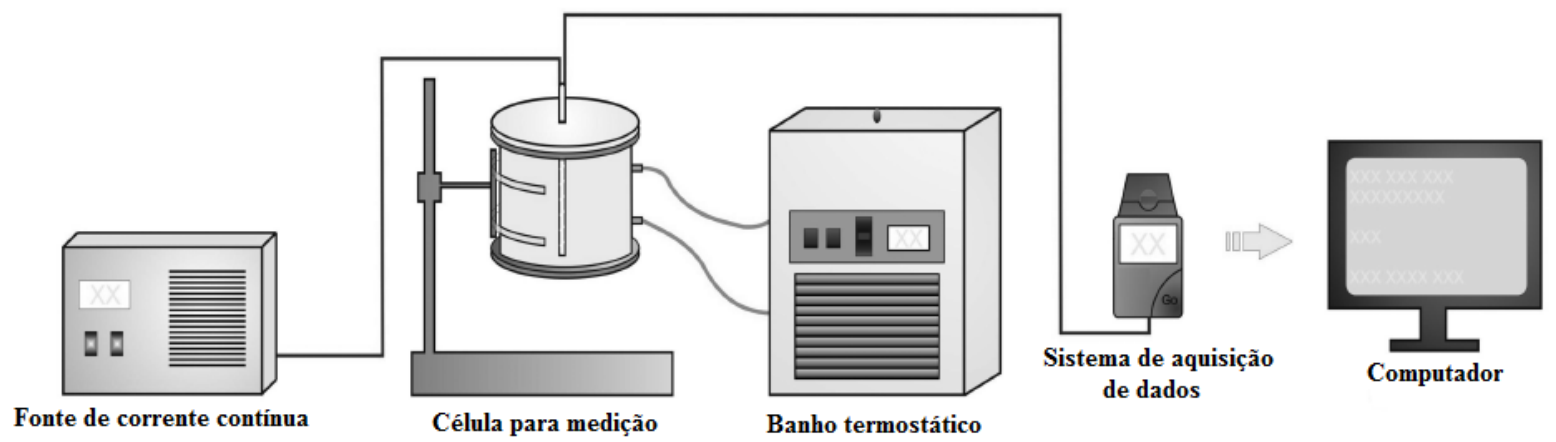

Tratando-se de materiais rígidos e de geometria bem definida, os ensaios para determinação de $\mathrm{K}_{0}$ não foram realizados em condições específicas de empacotamento.

\subsection{Método de cálculo}

$\mathrm{O}$ cálculo de $\mathrm{K}_{0}$ baseia-se na equação geral da condução de calor quando considerado escoamento unidirecional do fluxo de calor, conforme apresentado na Equação 1 considerando-se um aumento de temperatura em um intervalo de tempo $t_{2}-t_{1}$. Neste método, admite-se que, se energia é dissipada ao longo de uma direção através de um sólido a uma taxa constante, os aumentos de temperatura com o tempo são função apenas da fonte de energia e das propriedades do próprio sólido (McTaggart; Underwood, 1960).

$$
T_{2}-T_{1}=\frac{\dot{q} \cdot \ln \left(t_{2} / t_{1}\right)}{4 \pi K_{0}}
$$

onde $T_{2}-T_{1}$ corresponde a uma diferença de temperatura em intervalo de tempo $t_{2}-t_{1}$, provocado pelo fornecimento de fluxo de calor $\dot{q}$ constante ao sistema.

Os resultados obtidos experimentalmente para $\mathrm{K}_{0}$ de leitos porosos foram comparados com valores calculados a partir do modelo de resistências em paralelo sugerido por Goedeken et al. (1998) e mostrado na Equação 2.

$$
K_{0}=(1-\varepsilon) K_{s}+\varepsilon K_{a r}
$$

onde $\varepsilon$ é a porosidade do leito, $\mathrm{K}_{\mathrm{s}}$ é a condutividade térmica molecular da fração sólida e $\mathrm{K}_{\mathrm{ar}}$ é a condutividade térmica do ar. As propriedades do sólido e do ar foram extraídas de Perry e Chilton (1973).

\section{RESULTADOS E DISCUSSÃO}


Na Tabela 1 estão apresentados os resultados experimentais obtidos para $\mathrm{K}_{0}$ de sistemas monodispersos compostos por esferas de vidro e esferas de alumina em diferentes temperaturas. Nesta tabela, letras diferentes representam diferença significativa a $p \leq 0.05 \mathrm{em}$ tratamentos com diferentes temperaturas e letras maiúsculas e minúsculas representam tratamentos empregando-se diferentes partículas.

Tabela 1 - Valores para condutividade térmica efetiva na estagnação $\left(\mathrm{K}_{0}\right)$ de leitos de partículas esféricas determinados experimentalmente em diferentes temperaturas pelo método da sonda linear, em $\mathrm{W} / \mathrm{m} / \mathrm{K}$.

\begin{tabular}{|c|c|c|c|}
\hline Tipo de partícula & \multicolumn{3}{|c|}{ Temperatura $\left({ }^{\circ} \mathbf{C}\right)$} \\
\cline { 2 - 4 } constituinte do leito & $\mathbf{2 5}$ & $\mathbf{3 7 . 5}$ & $\mathbf{5 0}$ \\
\hline Esferas de vidro $(3 \mathrm{~mm})$ & $0.33 \pm 0.02^{\mathrm{a}}$ & $0.33 \pm 0.01^{\mathrm{a}}$ & $0.40 \pm 0.01^{\mathrm{b}}$ \\
\hline Esferas de alumina $(3 \mathrm{~mm})$ & $0.53 \pm 0.01^{\mathrm{A}}$ & $0.55 \pm 0.02^{\mathrm{A}}$ & $0.60 \pm 0.02^{\mathrm{B}}$ \\
\hline
\end{tabular}

$\mathrm{O}$ valor de $\mathrm{K}_{0}$ obtido a $50^{\circ} \mathrm{C}$ apresentou diferença significativa para as duas partículas analisadas em relação às demais temperaturas, o que indica que a elevação de $\mathrm{K}_{0}$ não necessariamente pode ser entendida como característica da composição química, mas está diretamente relacionada com mudanças de temperatura. Dos dados obtidos, sujeitos a um ajuste polinomial de segunda ordem, é possível se obter relações para $\mathrm{K}_{0}$ em função da temperatura, apresentadas nas Equações (3) e (4) para leitos de esferas de vidro e esferas de alumina, respectivamente.

$$
\begin{aligned}
& K_{0}=0,0002 T^{2}-0,014 T+0,54 \\
& K_{0}=0,0001 T^{2}-0,0044 T+0,58
\end{aligned}
$$

A literatura reporta valores para $\mathrm{K}_{0}$ de leitos de esferas de vidro próximos aos encontrados no presente trabalho. Tada et al. (2017) reportou $\mathrm{K}_{0}$ de um leito de esferas de vidro de $3 \mathrm{~mm}$ de diâmetro médio colocado em tambor horizontal parcialmente preenchido para ensaios de transferência de calor, correspondente a $0.33 \mathrm{~W} / \mathrm{mK}$. O mesmo valor foi relatado por Masamune e Smith (1963) para o mesmo conjunto de partículas.

Perazzini (2014) determinou $\mathrm{K}_{0}$ de leitos de esferas de alumina úmidas com diferentes diâmetros médios de partícula, $2.8 \mathrm{~mm}$ e $3.35 \mathrm{~mm}$, utilizando o método da sonda linear. Os valores encontrados foram de $8.4 \mathrm{~W} / \mathrm{mK}$ e $4.6 \mathrm{~W} / \mathrm{mK}$, respectivamente. Ensaios similares com estas partículas umidificadas até a saturação também foram realizadas no presente trabalho, obtendo-se valor médio de $3.44 \mathrm{~W} / \mathrm{mK}$ a $25^{\circ} \mathrm{C}$, próximo ao reportado pelo autor. Entretanto, foi evidenciada a migração de umidade durante os ensaios através de gotículas de vapor d'água condensadas na extremidade axial superior. Este fato contesta os resultados obtidos para tais partículas umidificadas, pois a análise do comportamento do sistema em relação à transferência de calor foi prejudicada pelo fenômeno simultâneo de transferência de massa.

A estimativa de $\mathrm{K}_{0}$ para leitos de esferas de vidro e alumina preditos pelo modelo de resistências em paralelo está apresentada na Tabela 2. A porosidade do leito foi admitida 
como 0.38, conforme Tada et al. (2017). As propriedades do sólido foram extraídas de Perry e Chilton (1983) para vidro a $25^{\circ} \mathrm{C}$ e alumina fundida.

Tabela 2 - Valores para condutividade térmica efetiva na estagnação $\left(\mathrm{K}_{0}\right)$ de leitos de partículas esféricas, calculados a partir do modelo de resistências em paralelo.

\begin{tabular}{|c|c|}
\hline Tipo de partícula constituinte do leito & $\mathbf{K}_{\mathbf{0}, \text { predito }}$ \\
\hline Esferas de vidro $(3 \mathrm{~mm})$ & 0.28 \\
\hline Esferas de alumina $(3 \mathrm{~mm})$ & 0.63 \\
\hline
\end{tabular}

Os resultados preditos pelo modelo de resistências em paralelo foram considerados satisfatórios para temperaturas de operação mais amenas em relação aos dados experimentais. É importante ressaltar que este modelo não considera a dependência de $\mathrm{K}_{0}$ diretamente em relação à temperatura, pois esta dependência está implícita no valor de $\mathrm{K}_{\mathrm{s}}$ e $\mathrm{K}_{\mathrm{ar}}$ adotados para cálculo. Desse modo, diz-se que o modelo foi satisfatório para representar o parâmetro $\mathrm{K}_{0}$ dos leitos porosos analisados neste trabalho.

\section{CONCLUSÕES}

A condutividade térmica efetiva na estagnação para leito de esferas de vidro variou de 0.33 a $0.40 \mathrm{~W} / \mathrm{mK}$, para uma faixa de temperatura de 25 a $50^{\circ} \mathrm{C}$. Nesta mesma faixa, este parâmetro variou de 0.53 a $0.60 \mathrm{~W} / \mathrm{mK}$ para leito de esferas de alumina. Para ambos os casos, foi constatada diferença significativa no valor do parâmetro quando determinado a $50^{\circ} \mathrm{C} \mathrm{em}$ relação às demais temperaturas. Os valores para condutividade térmica efetiva do leito preditos pelo modelo de resistências em paralelo apresentaram proximidade com os dados obtidos experimentalmente através do método da sonda linear.

\section{REFERÊNCIAS}

BOATENG, A. A.; BARR, P. V. A thermal model for the rotary kiln including heat transfer within the bed. Int. J. Heat and Mass Transfer, v. 39, p. 2131-2147, 1996.

CASCIATORI, F. P.; BÜCK, A.; THOMÉO, J. C.; TSOTSAS, E. Two-phase and twodimensional model describing heat and water transfer during solid-state fermentation within a packed bed bioreactor. Chem. Eng. J., v. 287, p. 103-116, 2016.

CASCIATORI, F. P.; LAURENTINO, C. L.; LOPES, K. C. M.; SOUZA, A. G. de; THOMÉO, J. C. Stagnant effective termal conductivity of agro-industrial residues for solid-state fermentation. Intern. J. of Food Properties, v. 16, p. 1578-1593, 2013.

CHOI, Y.; OKOS, M. R. Physical and Chemical Properties of Food. Martin R. Okos (Ed.). ASAW, St. Joseph, MI, p. 35-77, 1986.

FANAEI, M. A.; VAZIRI, B. M. Modeling of temperature gradientes in packed-bed solidstate bioreactors. Chem. Eng. Processing: Process Intensification, v. 48, p. 446-451, 2009. 
GOEDEKEN, D. L.; SHAH, K. K.; TONG, C. H. True thermal conductivity determination of moist porous food materials at elevated temperatures. J. Food Scienc., v. 63, p. $1062-$ 1063, 1998.

MASAMUNE, S..; SMITH, J. M. Thermal conductivity of beds of spherical particles. Industr. Eng. Chem. Fundam., v. 2, 1963.

McTAGGART, R.B.; UNDERWOOD, W.M. The termal conductivity of several plastics, measured by an unstead state method. Chem. Eng. Prog. Symp. Series, v. 56, p. 261268, 1960.

NECKEL, V. J.; MARIANI, V. C. Modelagem do congelamento da beterraba. Anais de Mecánica Computacional Vol XXIX, p. 5729-5740, 2010.

PERAZZINI, H. Secagem de sólidos porosos granulares. 2014. 189 f. Tese (Doutorado em Engenharia Química) - Centro de Ciências Exatas e de Tecnologia, Universidade Federal de São Carlos, São Carlos, 2014.

PERRY, R. H.; CHILTON, C. H. Chemical Engineer's Handbook. 5 ed. McGraw-Hill, 1973.

TADA, É. F. R.; BÜCK, A.; CASCIATORI, F. P.; TSOTSAS, E.; THOMÉO, J. C. Investigation of heat transfer in partially filled horizontal drums. Chem. Eng. Journal, v. 316, p. 988-1003, 2017.

THOMÉO, J. C. Análise experimental dos efeitos de entrada térmicos sobre os coeficientes de transferência de calor em leito fixo. 1990. 117 f. Dissertação (Mestrado em Engenharia Química) - Centro de Ciências e Tecnologia, Universidade Federal de São Carlos, São Carlos, 1990.

THOMÉO, J. C.; FREIRE, J. T. Transferência de calor em leitos fixos: avanços a partir da década de 90. In: Fenômenos de transporte em sistemas particulados. J. T. Freire, Ed. Suprema, 2009.

TSOTSAS, E.; MARTIN, H. Thermal-conductivity of packed-beds - a review. Chem. Eng. Process., v. 22, p. 19-37, 1987.

WANG, E. Q.; LI, S. Z.; TAO, L.; GENG, X.; LI, T. C. Modeling of rotating drum bioreactor for anaerobic solid-state fermentation. Appl. Energy, v. 87, p. 2839-2845, 2010. 